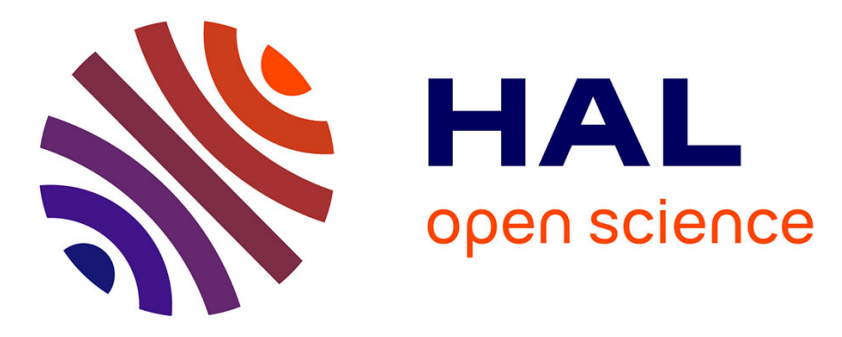

\title{
Effects of burst mode on transparent materials processing
}

\author{
C. Javaux Léger, K Mishchik, O Dematteo-Caulier, Stefan Skupin, B Chimier, \\ G Duchateau, A Bourgeade, C Hoenninger, E Mottay, J Lopez, et al.
}

\section{- To cite this version:}

C. Javaux Léger, K Mishchik, O Dematteo-Caulier, Stefan Skupin, B Chimier, et al.. Effects of burst mode on transparent materials processing. Laser-based Micro- and Nanoprocessing IX, Feb 2015, San Francisco, United States. pp.93510M, 10.1117/12.2079677 . hal-01133734

\section{HAL Id: hal-01133734 \\ https://hal.science/hal-01133734}

Submitted on 20 Mar 2015

HAL is a multi-disciplinary open access archive for the deposit and dissemination of scientific research documents, whether they are published or not. The documents may come from teaching and research institutions in France or abroad, or from public or private research centers.
L'archive ouverte pluridisciplinaire HAL, est destinée au dépôt et à la diffusion de documents scientifiques de niveau recherche, publiés ou non, émanant des établissements d'enseignement et de recherche français ou étrangers, des laboratoires publics ou privés. 


\title{
Effects of burst mode on transparent materials processing
}

\author{
C. Javaux Léger*a , K. Mishchik ${ }^{\mathrm{b}}$, O. Dematteo-Caulier ${ }^{\mathrm{b}}$, S. Skupin ${ }^{\mathrm{b}}$, B. Chimier ${ }^{\mathrm{b}}$, G. Duchateau ${ }^{\mathrm{b}}$, \\ A. Bourgeade ${ }^{\mathrm{b}}$, C. Hoenninger ${ }^{\mathrm{c}}$, E. Mottay ${ }^{\mathrm{c}}, \mathrm{J}_{\text {. Lopez }}^{\mathrm{b}}, \mathrm{R} \mathrm{Kling}^{\mathrm{a}}$ \\ ${ }^{a} \mathrm{ALPhANOV}$, Rue François Mitterand, 33400 Talence, France; \\ ${ }^{b}$ UNIV BORDEAUX CNRS CEA, CELIA UMR 5107, 33405 Talence, France; \\ 'AMPLITUDE SYSTEMES, 11, avenue de Canteranne, Cité de la Photonique, 33600 Pessac, \\ France.
}

\begin{abstract}
We investigated the effect of burstmode with nanosecond (ns) time delay between subpulses on sodalime glass volume machining. We observed in tight focusing configuration that the use of burstmode with ns time delay between subpulses does not increase the absorption efficiency and does not bring a significant effect on the heat affected zone diameter with respect to single pulse mode. On the contrary in loose focusing configuration the use of burst mode allows increasing the aspect ratio of the heat affected zone without extra energy absorption. This effect is highly interesting for filamentation glass cutting applications.
\end{abstract}

Keywords: ultrafast laser, burst, transparent material, welding, cutting, filamentation.

*clementine.javaux@alphanov.com; phone+33 (0)5 245452 17; www.alphanov.com

\section{INTRODUCTION}

In recent years, transparent materials such as glass, quartz, sapphire and others are more and more used for mass markets such as smart-electronics, optoelectronics or watchmaking. However conventional mechanical glass processing techniques do not provide full satisfaction in terms of speed, throughput and quality. Indeed these techniques often introduce side and detrimental effects such as residual stresses or crack formation (chipping). Therefore, there is a room for improvement and lasers have a role to play, especially ultrafast lasers since they have the capacity to perform involume processing for waveguide inscription ${ }^{1}$, for welding ${ }^{2}$, for cutting $^{3}$ or for midrodrilling ${ }^{4,5}$ for instance.

Glass welding by ultrafast laser irradiation is based on a localized melting at the interface between two glass substrates and a subsequent resolidification, which leads to a strong and permanent bonding. The bonding energy increases with the relative molten area ${ }^{6,7}$. This application usually requires tight focusing although the welding is also possible with a filament beam shaping ${ }^{8}$. In tight focusing conditions, the modification morphology has a comet-like shape with two embedded structures. The inner one corresponds to the laser photoexcited volume, meanwhile the outer one is directly related to the heat affected zone (HAZ) due to heat accumulation at high repetition rate, typically above $100 \mathrm{kHz}$. Composition changes in both inner and outer regions have been also reported ${ }^{10,11}$. Extend of HAZ increases with pulse energy and repetition rate $^{2}$.

Burstmode is also an option that must be considered. However the effect of burstmode on laser-dielectric materials interaction highly depends on the delay between subsequent pulses in the burst (called thereafter subpulses). Indeed, if the delay between subpulses is shorter than the mean cooling time of photoexcited electrons (typically few tens of ps), the burstmode contributes to enhance the laser-matter coupling and enlarge the HAZ diameter as described by Sugioka et $\mathrm{al}^{12}$. On the other hand, if the delay between subsequent pulses in the burst is longer, the laser-matter interaction does not benefit from burstmode. Indeed on the ns time scale most of the photoexcited electrons have relaxed, and although the irradiated area has not yet returned to room temperature, the HAZ diameter has been shown to become smaller than in single pulse $\operatorname{mode}^{12}$.

Laser-based glass cutting is also a subject of high interest from an industrial point of view. Ablation cutting is quite slow and produces a lot of dust. Another technique is scribe and break but the main drawback is an uncontrolled path deviation along the sidewall (cut plan). In comparison to these previous techniques, filamentation cutting offers several 
advantages such as zerokerf, dustfree, smooth and regular cut plan, and rapid processing (several $100 \mathrm{~mm} / \mathrm{s}$ ). Filamentation cutting consists in weakening the glass by producing an elongated modification along the glass thickness as a precutting step, and then in releasing the two parts thanks to a mechanical pressure ${ }^{3}$. The filament is produced at high laser intensity, in specific focusing conditions, and results from equilibrium between self-focusing (Kerr effect) and defocusing when the intensity overcomes the critical power ${ }^{4}$. The filament may propagate the laser beam energy on mmlong distance. The effect of burstmode with ns-delay on filament morphology has been investigated by Esser and Rezaei $^{4,5}$. They have shown that burstmode contributes to create a filament, which may act as a waveguide for subsequent high aspect ratio microdrilling in dielectric materials.

In this paper we report on the influence of burstmode with a ns-delay on the heat affected zone morphology while focusing a femtosecond laser beam inside sodalime glass. The aim is to improve glass welding on one hand, and filamentation cutting on the other hand. The results will be compared to single pulse processing and discussed in terms of HAZ diameter, HAZ length and nonlinear absorptivity. We have demonstrated that burstmode with a ns-delay is useless for glass welding since it does not enlarge the HAZ, whereas it is useful for filamentation cutting since it contributes to extend the length of the filament for a same level burst energy.

\section{EXPERIMENTAL SETUP}

For our experiments we used a Satsuma Yb-doped fiber femtosecond laser from Amplitude Systemes, emitting at 1030 $\mathrm{nm}$ with pulse duration down to $300 \mathrm{fs}$ and with repetition rate varying from $500 \mathrm{kHz}$ to $2 \mathrm{MHz}$. Its output power is $5 \mathrm{~W}$ and the maximal output pulse energy varies from $2.5 \mu \mathrm{J}$ to $10 \mu \mathrm{J}$. The laser can be used in single pulse mode as well as in burstmode (schematized on Fig. 1a). The delay between subpulses in the burst is defined by the oscillator repetition rate, which is of $40 \mathrm{MHz}$ leading to a time delay of $25 \mathrm{~ns}$. It is thereafter referred to as $25 \mathrm{~ns}$-burstmode. The number of subpulses in the burst can be tuned from 1 (single pulse mode) to 10 . It is commonly referred to as pulses per burst (PPB). The energy $E_{p}$ of the burst (sum of the energies of the subpulses) is equal to the energy of the pulse in single pulse mode.

a.
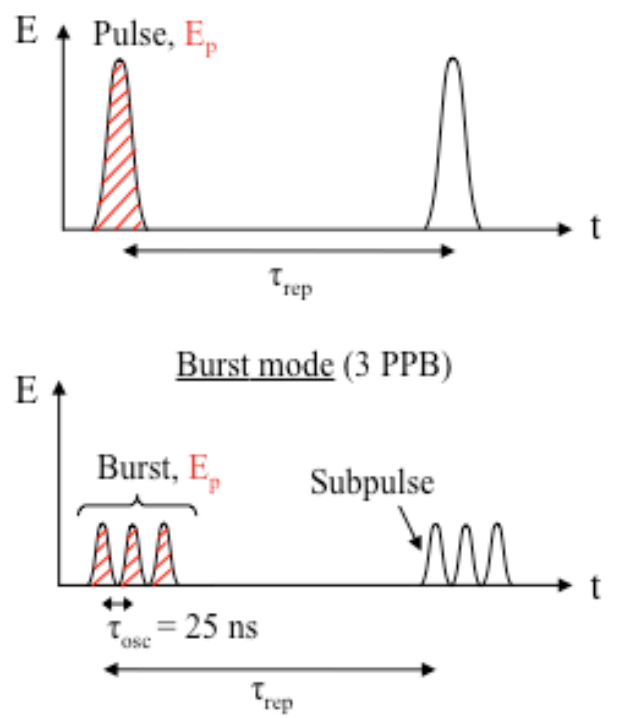

b.
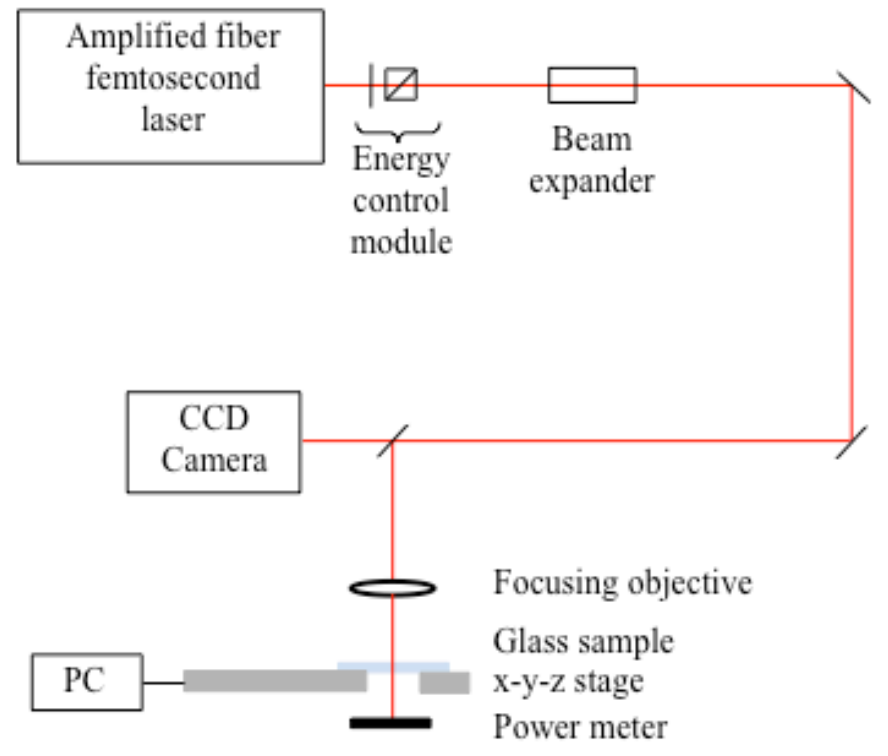

Figure 1. a Schematic representation of the burstmode.. b. Schematic representation of the experimental setup. The energy control module is composed of a half-wave plate and a polarizing beam splitter cube.

The experimental setup used for this study is presented in Figure 1b. It included the laser, a half-wave plate combined with a polarizer cube to control the pulse energy, a beam expander, an inverted microscope (Olympus, Eclipse) equipped with a microscope objective (Mitutoyo, M Plan APO NIR) to focalize the laser beam on the sample, and a XYZ motorized stages system (Aerotech, A3200) with a specific sample holder for positioning the sample under laser beam. We used three objectives successively in this study with a magnifying power of $20 x, 10 x$ and $5 x$ respectively. Taking into account the beam diameter on the entrance pupil of the objectives, the effective used numerical apertures (NA) were 
$0.4,0.26$ and 0.08 respectively. When using 0.4 NA the setup is said to be in tight focusing configuration, and when using 0.26 or 0.08 NA it is said to be in moderate and loose focusing configurations respectively. The glass samples were commercially available $1 \mathrm{~mm}$-thick sodalime microscope cover slides (Duran Group). We used the CCD camera to localize the sample surface and then the laser beam was focalized $250 \mu \mathrm{m}$ deep in the glass volume.

We speak about experiments in static mode when the laser beam was focalized into the sample at a fixed position for 10,000 pulses. We speak about experiments in dynamic mode when the beam was scanned over the sample at constant speed. The number of equivalent pulses per point of irradiation is calculated taking into account the scanning velocity, the repetition rate and the interaction volume diameter. In order to be close to the static mode with 10,000 pulses per fixed position, we used a scanning speed of $5 \mathrm{~mm} \cdot \mathrm{s}^{-1}$. The dynamic configuration allowed also the absorption measurements using a power meter measuring the time-averaged transmitted power through the glass sample during laser beam scanning the sample. Assuming reflection and scattering from the laser induced plasma are negligible the nonlinear absorptivity is given by $A=1-\frac{P_{t}}{P_{i}} \frac{1}{(1-R)^{2}}$, where Pi is the incident laser power, Pt the transmitted laser power through the glass sample and $\mathrm{R}$ the Fresnel reflectivity.

The HAZ in the glass after laser irradiation can be observed either from the laser incident direction (called top view), or perpendicularly to the incident laser beam such that the observed HAZ is composed of the superposition of the heat affected zones generated by the laser scanning (called side view). The sample characterization was made with an optical measurement microscope (Mitutoyo, MF-A1010H) coupled with a QM-Data 200 calculator, using 10x or 20x objectives.

\section{RESULTS AND DISCUSSION}

\subsection{Burstmode influence on HAZ diameter for glass welding application}

The bonding strength of a welding seam is linked to the absorption efficiency of the laser beam at the interface of the glass samples. When the absorption is more efficient, the HAZ is wider, and the strength of the joint is higher ${ }^{6,7}$. In order to evaluate the interest of burstmode on glass welding, we studied the evolution of the glass absorption efficiency when using the burstmode within tight focusing configuration (NA 0.4). To this aim we irradiated the sodalime glass sample in static mode varying the number of PPB from 1 to 5 , for repetition rate varying from $2 \mathrm{MHz}$ down to $250 \mathrm{kHz}$. The resulting material modifications observed by optical microscopy are reported on figure 2 .

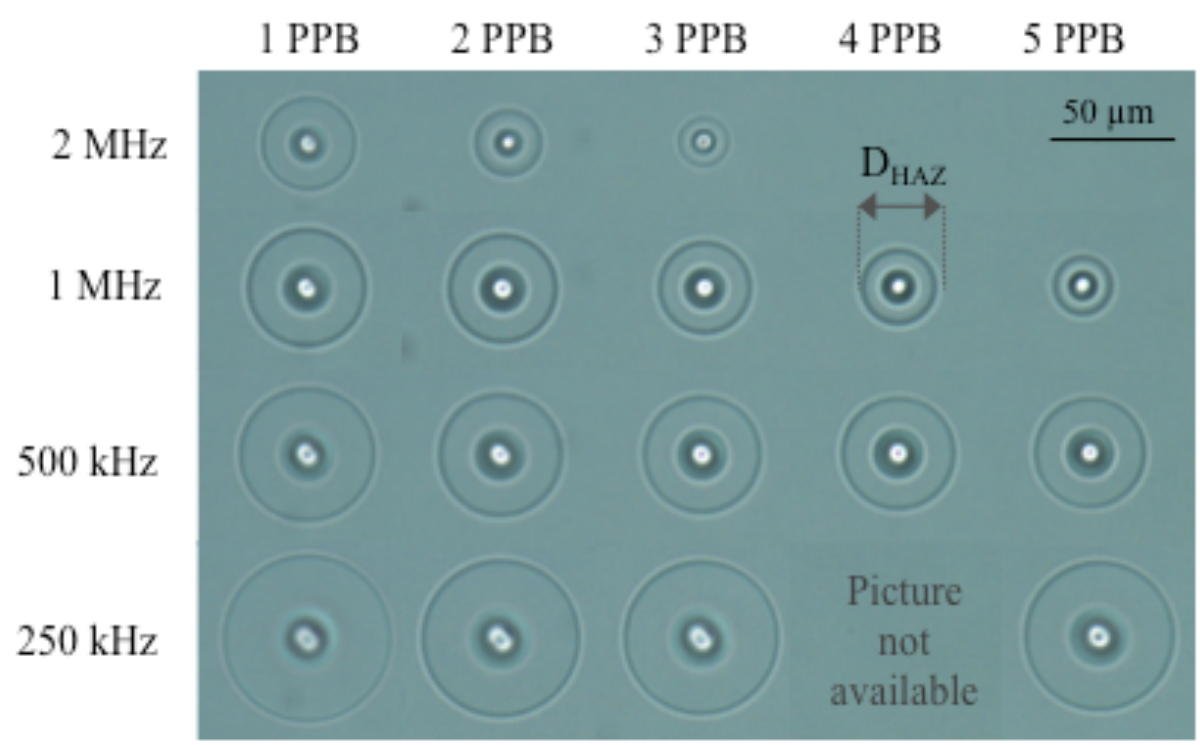

Figure 2. Top view optical microscopy observation of the HAZ created in the glass sample for 1 to 5 PPB and repetition rate from $2 \mathrm{MHz}$ down to $250 \mathrm{kHz}$. The focusing laser beam has a numerical aperture of 0.4 (objective 20x) and its incidence is perpendicular to the plane of the picture. Each HAZ was irradiated by 10,000 pulses in static configuration. The average power available on target was $500 \mathrm{~mW}$ for each repetition rate. The target pulse energy was $0.25,0.5,1$ and $2 \mu \mathrm{J}$ for $2 \mathrm{MHz}$, $1 \mathrm{MHz}, 500 \mathrm{kHz}$ and $250 \mathrm{kHz}$ respectively. 
Figure 2 shows the influence of the number of PPB on the HAZ diameter for repetition rates of $2 \mathrm{MHz}, 1 \mathrm{MHz}, 500 \mathrm{kHZ}$ and $250 \mathrm{kHz}$. For each repetition rate the target power is kept constant and fixed at $500 \mathrm{~mW}$. We observe that the HAZ is always composed of two concentric circular zones. The inner circular zone corresponds to the laser photoexcited volume and the outer zone to the molten HAZ as reported by Shimizu et $\mathrm{al}^{13}$. When the glass sample is irradiated in single pulse mode (1 PPB), the extent of the HAZ increases when the laser repetition rate decreases. Since the target power is kept constant when varying the repetition rate, a decrease of the repetition rate induces an increase of the target pulse energy. The results observed here are thus consistent with respect to previous published results ${ }^{2}$. At each fixed number of PPB, from 2 to 5, the HAZ diameter also varies inversely to the repetition rate since the subpulses energy is higher when the repetition rate decreases. The measured values of the HAZ diameter with respect to the number of PPB for each repetition rate are reported on the graph of figure 3.

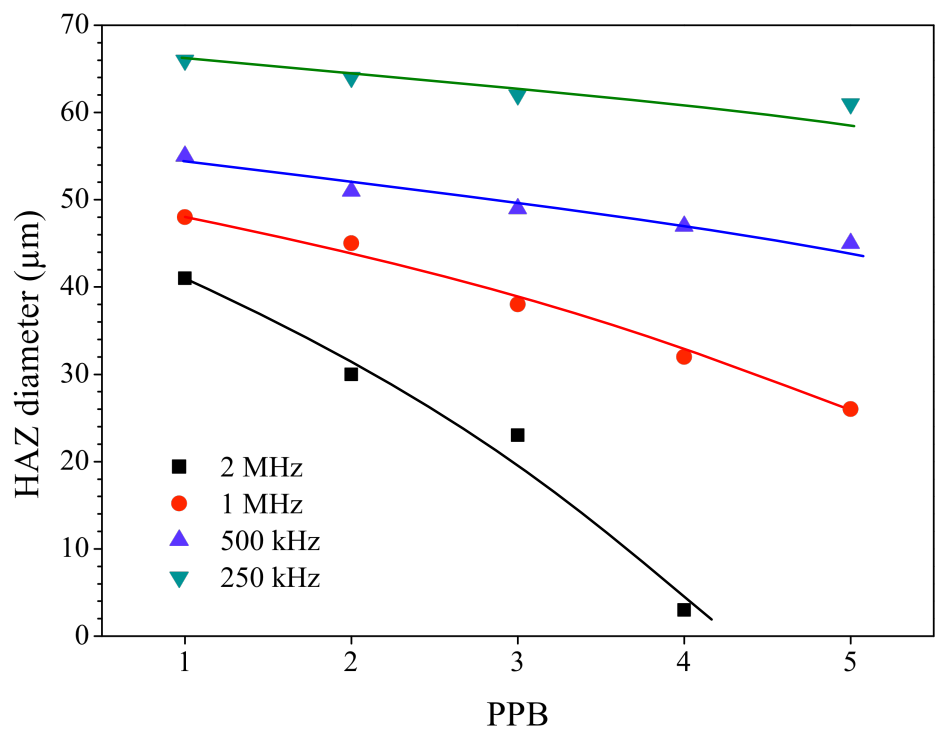

Figure 3. Measurement of the diameter of the HAZ shown on figure 2 with respect to the number of PPB. The data are underlined with spline fits.

At a fixed repetition rate, we observe a decrease of the diameter of the HAZ for higher numbers of PPB. This result is in accordance with the paper published by $\mathrm{S}$. Wu et $\mathrm{al}^{14}$. As mentioned above, they demonstrated that the effect of doublepulse ultrasfast laser irradiation was linked to the delay time between the two subpulses. They reported that the HAZ becomes smaller than for single pulse mode for a time delay between sub-pulses beyond some tenth of ns, which is consistent with our 25 ns delay time burstmode.

We also measured the absorption of the laser beam induced by the laser-glass interaction while varying the number of PPB. Figure 4 reports the absorption measurements for 1 to $5 \mathrm{PPB}$ and for repetition rates going from $500 \mathrm{kHz}$ to 2 MHz. The average power available on target was still kept at $500 \mathrm{~mW}$ for each repetition rate. In single pulse mode, the absorption increases when the repetition rate decreases, in accordance with the HAZ size. Indeed the increase of pulse energy leads to stronger multiphoton absorption, and therefore to larger HAZ. For each repetition rate, the glass nonlinear absorptivity appears to decrease while increasing the number of PPB. This result is also consistent with the HAZ diameter measurements. When using the burstmode at constant pulse energy, the subpulses energy decreases with the number of PPB. Since after 15 ns most of the photoexcited electrons have relaxed, the use of burstmode only induces a decrease of the pulse energy and thus of the nonlinear absorption. A laser with ns-burstmode is therefore not adapted to address glass welding application. 


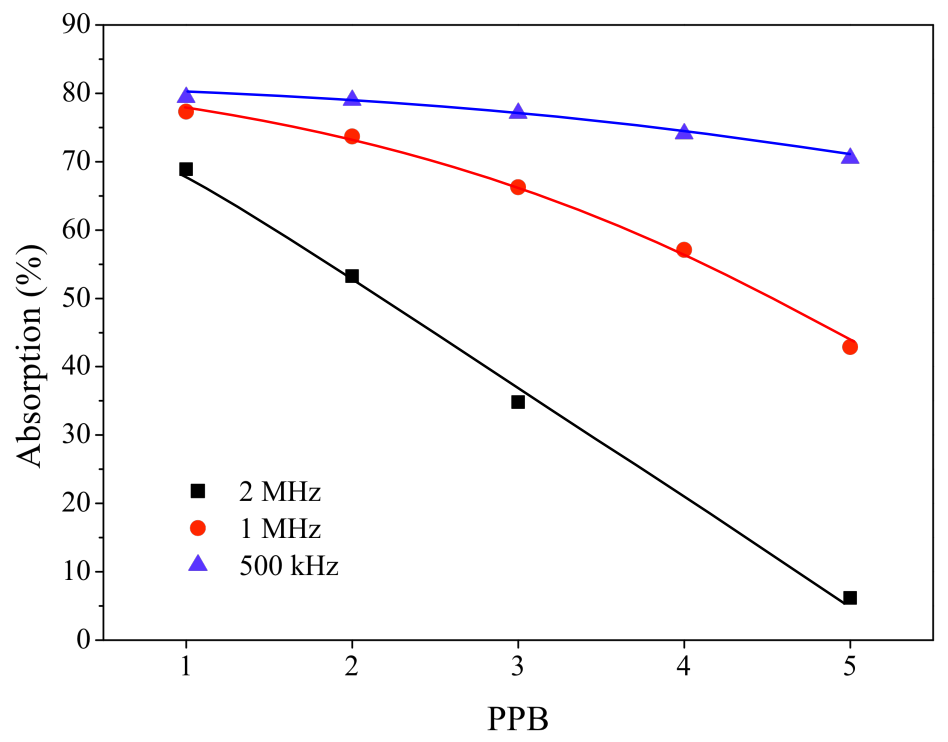

Figure 4. Absorption efficiency of the intravolume interaction versus the number of PPB. The measurements were done in dynamic configuration at $5 \mathrm{~mm} \cdot \mathrm{s}^{-1}$. The average power available on target was $500 \mathrm{~mW}$ for each repetition rate. The target pulse energy was $0.25,0.5,1$ and $2 \mu \mathrm{J}$ for $2 \mathrm{MHz}, 1 \mathrm{MHz}, 500 \mathrm{kHz}$ and $250 \mathrm{kHz}$ respectively. The data are underlined with spline fits.

\subsection{Burstmode influence on filament length for glass (pre)cutting application}

Then we investigated the interest of using ns-burstmode for filamentation. Figure 5 shows the influence of PPB on the HAZ morphology for moderate (NA 0.26) and loose (NA 0.08) focusing configurations for static mode glass irradiation.

a.

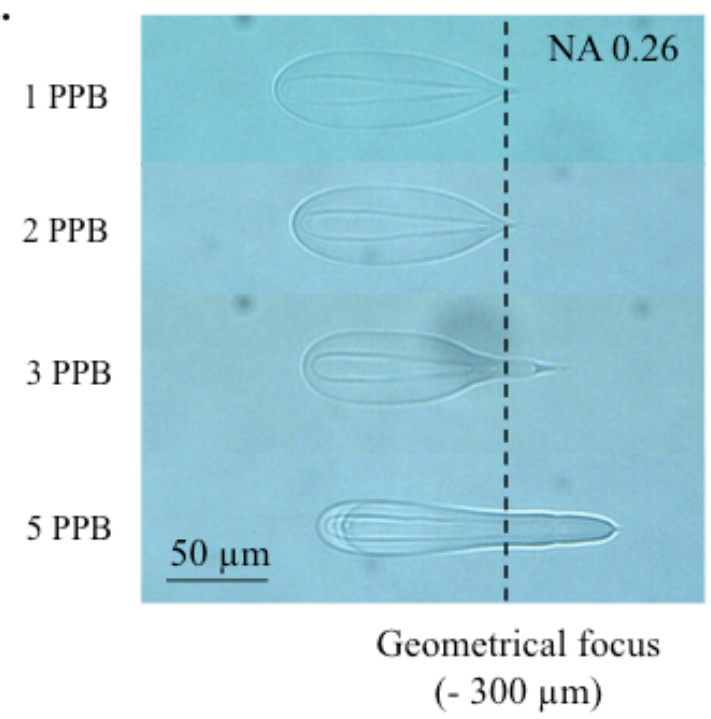

b.

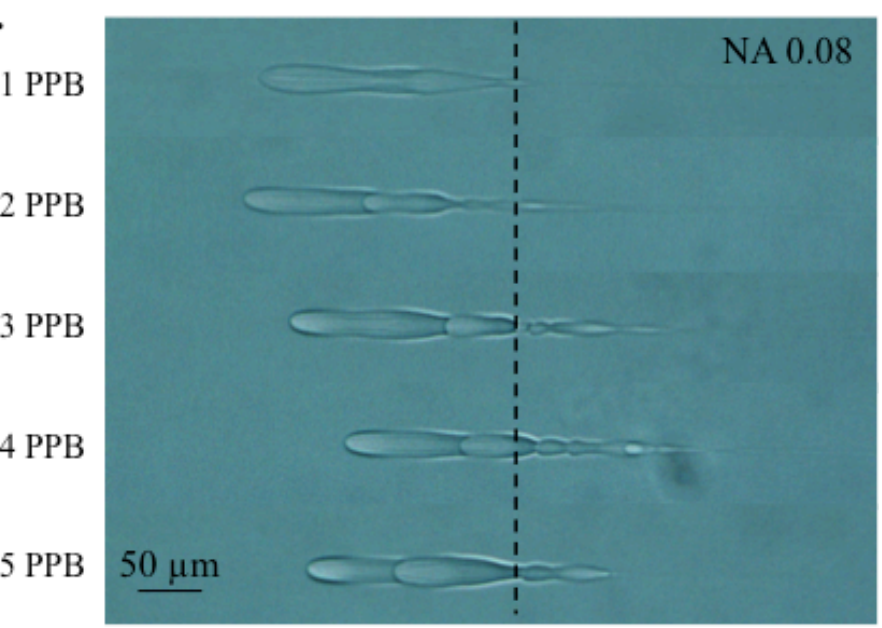

Geometrical focus

$(-450 \mu \mathrm{m})$

Figure 5. Side view optical microscopy observation of the HAZ created in the glass samples for moderate (NA 0.26) and loose (NA 0.08) focusing configurations in static mode. The laser is incident on the glass sample from the left of the picture. a. The target power was $1.1 \mathrm{~W}$, the pulse duration was $380 \mathrm{fs}$, the repetition rate was $500 \mathrm{kHz}$, and each $\mathrm{HAZ}$ was created by irradiation of 500 successive pulses. b. The target power was $1.5 \mathrm{~W}$, the pulse duration was $300 \mathrm{fs}$, the repetition rate was $500 \mathrm{kHz}$, and each HAZ was created by irradiation of 1,000 successive pulses. 
With $0.26 \mathrm{NA}$ in single pulse mode, we observe that the material modification present a comet-like shape with a laser photoexcited zone surrounded with the molten HAZ zone as in the case of tight focusing ${ }^{13}$. The tail of the comet is localized on the geometrical focus position, and its front delimitation is positioned closer to the incident glass surface due to the optical Kerr effect. When increasing the PPB, the two embedded zones are still visible, however the aspect ratio of the HAZ increases. For 3 and 5 PPB the width of the HAZ decreases while its length increases. The front delimitation moves back towards the geometrical focus position for higher numbers of PPB because a diminution of the subpulses energy reduces the optical Kerr effect. The elongation of the HAZ follows the laser propagation direction, which indicates a better energy propagation efficiency.

In loose focusing configuration (0.08 NA), the material modification no longer has a comet shape, but rather looks like a filament. The HAZ is much thinner and longer with respect to higher NA. The filament is composed of a head, in front of the geometrical focus position, and a tail, which goes far beyond. We observe that the filament also lengthens when the number of PPB increases up to 3 and then decreases down to the size of the filament obtained in single pulse mode or even below. As for the moderate focusing configuration, the front delimitation of the glass modified zone appears deeper when the number of PPB increases up to 4, due to lower optical Kerr effect. For 5 PPB, we have no explanation concerning the position of the filament front delimitation. Figure 6 reports the observation of the filaments obtained in dynamic mode at velocity $5 \mathrm{~mm} \cdot \mathrm{s}^{-1}$, in order to enhance the contrast of the tail of the filament. The length of the whole HAZ can thus be evaluated more precisely. The measured lengths are reported on the graph of figure 7.

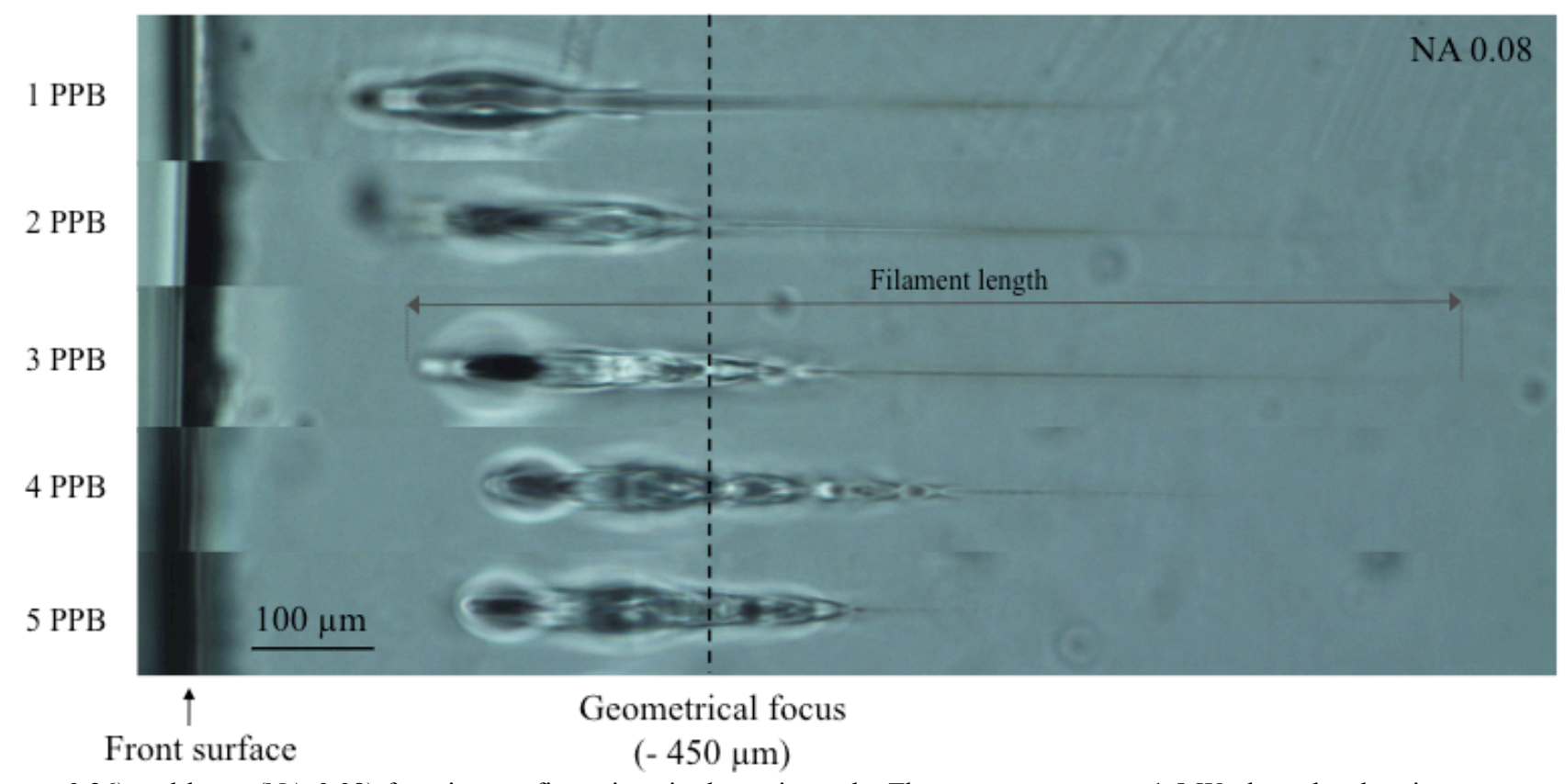

0.26 ) and loose (NA 0.08) focusing configurations in dynamic mode. The target power was $1.5 \mathrm{~W}$, the pulse duration was $300 \mathrm{fs}$, the repetition rate was $500 \mathrm{kHz}$, and each HAZ was created with translation velocity $5 \mathrm{~mm} \cdot \mathrm{s}^{-1}$.

Additional insights give us the influence of the pulse energies on the laser affected zone length in the burstmode irradiation. The results for four different target pulse energies are presented in figure 7. For pulse energies below $2.4 \mu \mathrm{J}$ the length of the generated filament decreases with the number of PPB. For higher energies available on target, the behavior alters. The use of burstmode interestingly generates longer filaments. The longest HAZ is obtained for 3 PPB for a burst energy of $3.1 \mu \mathrm{J}$ and $3.8 \mu \mathrm{J}$. The filament reaches a length of around $950 \mu \mathrm{m}$ and $1200 \mu \mathrm{m}$ for $3.1 \mu \mathrm{J}$ and 3.8 $\mu \mathrm{J}$ respectively, that is $200 \mu \mathrm{m}$ longer compared with single pulse mode for both pulse energies. 


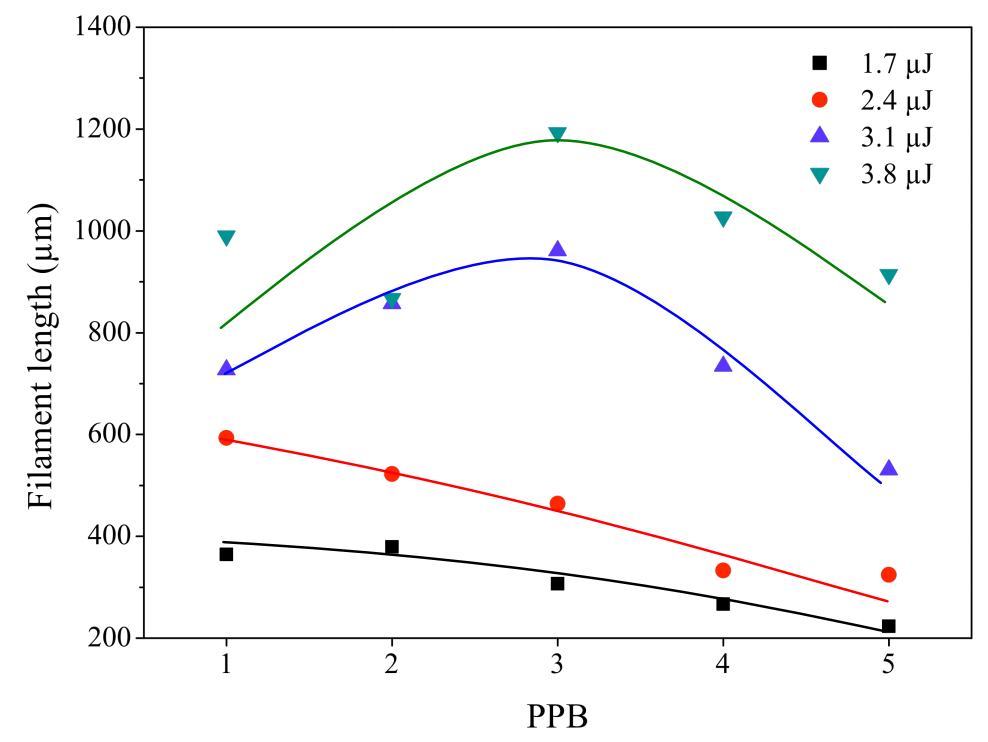

Figure 7. Measurement of the filament length according to experiments in loose focusing configuration and dynamic mode reported on figure 6 with respect to the number of PPB, at $500 \mathrm{kHz}$. The data are underlined with spline fits.

We then measured the absorption efficiency of the laser-glass interaction in loose focusing configuration for 1 to 5 PPB and for varying target energy. Figure 8 shows the influence of the number of PPB on the energy absorption in the sample for target energy varying from $1.6 \mu \mathrm{J}$ to $3.8 \mu \mathrm{J}$.

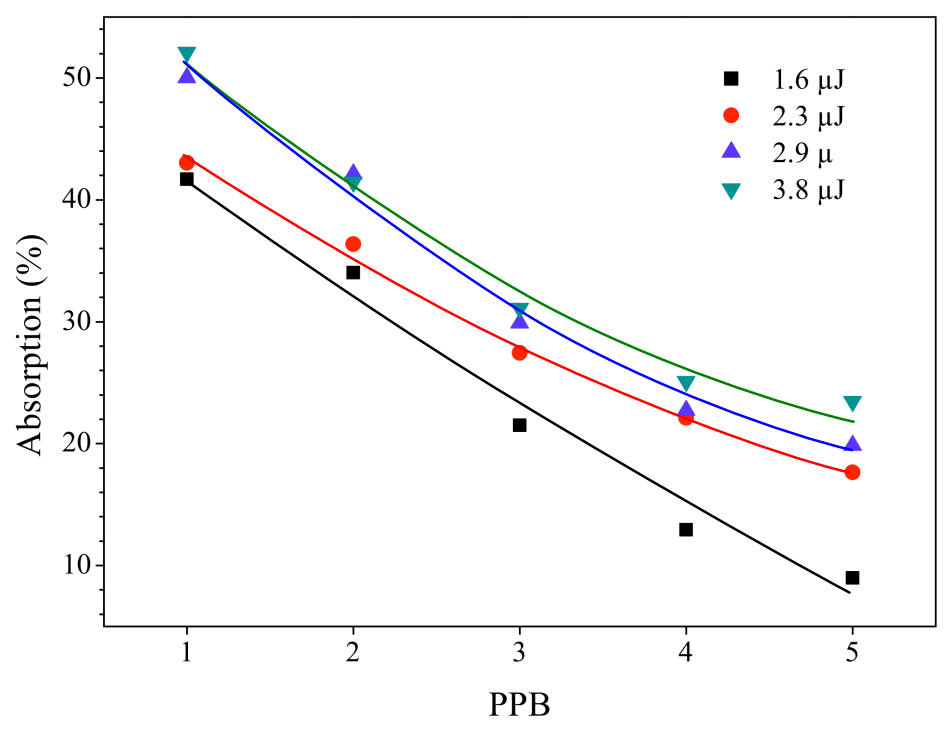

Figure 8. Measurement of the absorption efficiency versus the number of PPB at $500 \mathrm{kHz}$. The data are underlined with spline fits.

We observe that whatever the target energy the absorption efficiency decreases when the number of PPB increases. Consequently the use of burstmode in loose focusing configuration allows increasing the filamentation length and thus the energy propagation without extra energy absorption. This striking result is highly promising for filamentation glass cutting processes where the point is to generate filaments as long as possible. Single pass full cutting would require 
higher energy, but in present case the filament generation could be used as a precutting step. It would weaken the glass and generate mechanical stress along a predefined line, as required for cutting.

\subsection{Transient waveguiding effect}

Considering the results presented in the previous sections, we would like to focus on the particular filamentation regime realized in the burstmode. Despite the overall absorbed energy is lower, the filaments are longer. Moreover, longer filaments, which are caused by strongly nonlinear processes, are produced with the pulses having much lower peak intensity compared with convenient irradiation with single pulses. Apparently, the transient changes in electronic structure and structural modifications of the glass produced by the first subpulse strongly affect nonlinear propagation of the subsequent subpulses. Due to small time interval between the subpulses in the burst, relaxation processes have not finished yet and such transient changes are especially effective. Schematically these relaxation processes are illustrated on the Figure 9, where we present the characteristic times of the different steps of the light-matter interaction at high intensity in glasses.

Structural modification of the matter

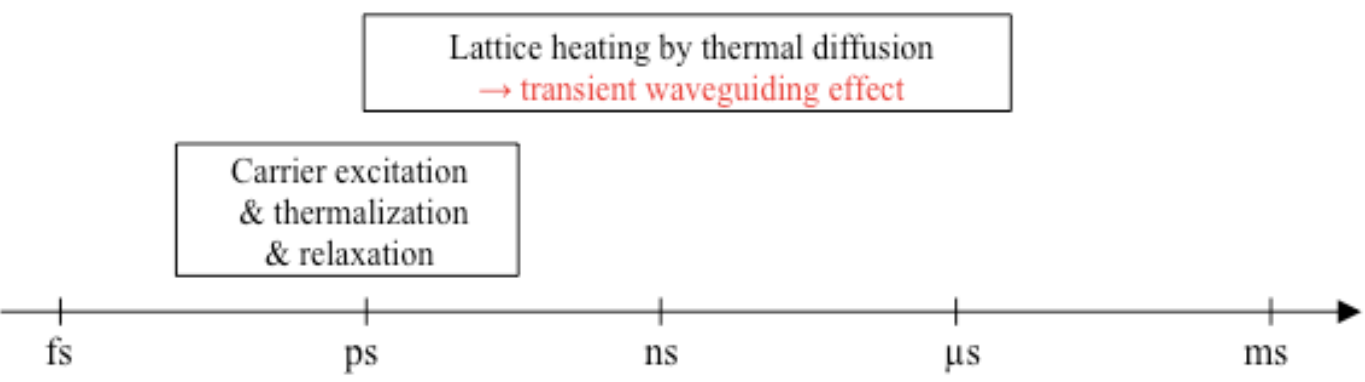

Figure 9. Schematic representation of light-matter interactions at high intensity versus time in sodalime glass.

Among others we selected the factors, which are non-negligible when the next subpulse arrives and which can significantly influence propagation of the subsequent pulse as reported in the literature. These factors are residual electrons in the conduction band ${ }^{14}$, changes of nonlinear susceptibility directly causing nonlinear propagation ${ }^{15,16}$, refractive index changes induced by the presence of the permanent defects ${ }^{16,17}$ and thermally induced variation of the refractive index ${ }^{18}$. Most of these factors, however, lead to mainly light defocusing or negligibly affects nonlinear propagation. On the contrary, thermal-induced variation of refractive index can produce substantial index variation and deserves more attention.

In our case, when the laser works at $500 \mathrm{kHz}$, the absorbed energy has not completely dissipated before the following burst arrives. In the long run this causes heat accumulation and gradual pulse-to-pulse temperature rise ${ }^{19,20}$. In the burst mode, the laser oscillator frequency is $40 \mathrm{MHz}$ so the time delay between subpulses is $25 \mathrm{~ns}$. At such short time scale heat diffusion processes have even less time to evacuate the absorbed energy. As a consequence the lattice temperature, which is subjected to sufficient local rise in the focal zone ${ }^{20}$, induces a bandgap collapse ${ }^{18}$. The main factor that determines the collapse of glass bandgap is the thermal vibrations rather than the structural disorder. Therefore the heatinduced bandgap changes follow the temperature increase almost instantly after 50-100fs (a time characteristic of lattice vibrations). This leads to a transient positive refraction index variation estimated at the order of 0.02 at the laser wavelength. On the other hand, the temperature increase of the material induces a volume expansion. This density variation leads to a decrease of the refractive index. The volume expansion, however, has different characteristic time, which is given by the viscoelastic relaxation $\tau_{\mathrm{s}}=\eta(\mathrm{T}) / \mathrm{G}$, where $\eta$ is the viscosity and $\mathrm{G}$ is the bulk modulus of sodalime glass. Considering a temperature close to the glass vitreous transition where the material is expected to be significantly modified, the volume expansion characteristic time lies in the range of hundreds of ns. Therefore we suggest that a competition between both the increase and decrease of the refractive index in the laser-material interaction zone may result in an overall positive index variation. This variation forms a transient focusing lens, leading to a transient waveguiding effect. The energy deposition zone thus elongates, favoring the filamentation phenomenon without extra energy absorption. This explanation is consistent with our experimental data, however it needs additional experimental confirmation supported by accurate theoretical simulations. 


\section{CONCLUSION}

We studied the effect of burstmode with 25 ns time delay on sodalime glass volume machining. We investigated primarily the influence of numerical aperture by comparing material modifications induced in single pulse or in burstmode regime. In tight focusing configuration the use of burstmode did not show any positive effect. Ns-bursts lasers are not ideal candidates for welding processes. On the contrary, in loose focusing configuration the burstmode with nsdelay time proved to be highly interesting. It increases drastically the HAZ aspect ratio and therefore the filamentation efficiency without extra energy needed. We proposed a thermal effects-based explanation for this observation, named a transient waveguiding effect. Further experimental work such as pump-probe measurements would allow verifying this hypothesis. This effect is promising for glass cutting processes at least for the filament precutting step. The optimization of the subpulses delay time would probably even allow to increase this waveguiding effect and thus the filament length.

\section{ACKNOWLEDGEMENTS}

We acknowledge the European Commission, the French Ministry of Industry and the Aquitaine Regional Council for support and funding.

\section{REFERENCES}

[1] Davis, K. M., Miura, K., Sugimoto, N., Hirao, K., "Writing waveguides in glass with a femtosecond laser.," Opt. Lett. 21(21), 1729-1731 (1996).

[2] Miyamoto, I., Cvecek, K., "Internal modification of glass by ultrashort laser pulse and its application to microwelding," 187-208 (2014).

[3] Kumkar, M., Bauer, L., Russ, S., Wendel, M., Kleiner, J., Grossmann, D., Bergner, K., Nolte, S., "Comparison of different processes for separation of glass and crystals using ultrashort pulsed lasers," Proc. SPIE, Vol. 9351, 897214 (2014).

[4] Esser, D., Rezaei, S., Li, J., Herman, P. R.., Gottmann, J., "Time dynamics of burst-train filamentation assisted femtosecond laser machining in glasses.,” Opt. Express 19(25), 25632-25642 (2011).

[5] Rezaei, S., "Burst-train generation for femtosecond laser filamentation-driven micromachining", thesis for degree of Master of Applied Science, Toronto University (2011).

[6] Alexeev, I., Cvecek, K., Schmidt, C., Miyamoto, I., Frick, T., Schmidt, M., "Characterization of shear strength and bonding energy of laser produced welding seams in glass," Proc. LPM, 1-5 (2012).

[7] Richter, S., Nolte, S., Tünnermann, A., "Ultrashort pulse laser welding - a new approach for high- stability bonding of different glasses," Physics Procedia 39, 556-562 (2012).

[8] Hélie, D., Lacroix, F., Vallée, R., "Reinforcing a Direct Bond between Optical Materials by Filamentation Based Femtosecond Laser Welding," Proc. LPM, 1-8 (2012).

[9] Shimizu, M., Sakakura, M., Ohnishi, M., Shimotsuma, Y., Nakaya, T., Miura, K., Hirao, K., "Mechanism of heat-modification inside a glass after irradiation with high-repetition rate femtosecond laser pulses," J. Appl. Phys. 108(7), 073533 (2010).

[10] Shimizu, M., Sakakura, M., Kanehira, S.., Nishi, M., "Formation mechanism of element distribution in glass under femtosecond laser irradiation,” Opt. Lett. 36(11), 2161-2163 (2011). 
[11] Sakakura, M., Yoshimura, K., Kurita, T., Shimizu, M., Shimotsuma, Y., Fukuda, N., Hirao, K.., Miura, K., "Condensation of Si-rich region inside soda-lime glass by parallel femtosecond laser irradiation," Opt. Express 22(13), 16493 (2014).

[12] Sugioka, K., Wu, S., Midorikawa, K., "Double-Pulsed Ultrafast Laser Welding of Glasses Toward Enhancement of Process Efficiency." Proc. SPLC, SLPC5-2 (2014).

[13] Shimizu, M., Sakakura, M., Ohnishi, M., Shimotsuma, Y., Nakaya, T., Miura, K., Hirao, K., "Mechanism of heat-modification inside a glass after irradiation with high-repetition rate femtosecond laser pulses," J. Appl. Phys. 108(7), 073533 (2010).

[14] Wu, S., Wu, D., Xu, J., Hanada, Y., Suganuma, R., Wang, H., Makimura, T., Sugioka, K., Midorikawa, K., "Characterization and mechanism of glass microwelding by double-pulse ultrafast laser irradiation.," Opt. Express 20(27), 28893-28905 (2012).

[15] Zoubir, A., Richardson, M., Canioni, L., Brocas, A., Sarger, L., "Optical properties of infrared femtosecond laser-modified fused silica and application to waveguide fabrication,” J. Opt. Soc. Am. B 22, 2138-2143 (2005).

[16] Royon, A., Rivero-Baleine, C., Zoubir, A., Canioni, L., Couzi, M., Cardinal, T., Fargin, E., Richardson, M., Richardson, K., "Evolution of the linear and nonlinear optical properties of femtosecond laser exposed fused silica,” J. Opt. Soc. Am. B 26(11), 2077-2083 (2009).

[17] Streltsov, A. M., Borrelli, N. F., "Study of femtosecond-laser-written waveguides in glasses," J. Opt. Soc. Am. B 19(10), 2496 (2002).

[18] Saito, K., Ikushima, A. J., “Absorption edge in silica glass,” PhysRevB.62, 8584-8587 (2000).

[19] Eaton, S., Zhang, H., Herman, P., Yoshino, F., Shah, L., Bovatsek, J., Arai, A., "Heat accumulation effects in femtosecond laser-written waveguides with variable repetition rate.," Opt. Express 13(12), 4708-4716 (2005).

[20] Richter, S., Zimmermann, F., Döring, S., Tünnermann, A., Nolte, S., "Ultrastable bonding of glass with femtosecond laser bursts," A. Heisterkamp, P. R. Herman, M. Meunier, and S. Nolte, Eds., 86111C - 86111C 8 (2013). 